\title{
Two-color phase control of high-order harmonic generation in intense laser fields
}

\author{
Dmitry A. Telnov, * Jingyan Wang, and Shih-I Chu \\ Department of Chemistry, University of Kansas, Lawrence, Kansas 66045
}

(Received 30 May 1995)

\begin{abstract}
We present a time-independent generalized Floquet approach for nonperturbative treatment of high-order harmonic generation (HG) in intense one- and two-color laser fields. The procedure consists of the following elements: (i) determination of the complex quasienergy eigenvalue and eigenfunction by means of the nonHermitian Floquet formalism, wherein the Floquet Hamiltonian is discretized by the complex-scaling generalized pseudospectral technique [Wang, Chu, and Laughlin, Phys. Rev. A 50, 3208 (1994)], and (ii) calculation of the HG rates based on the approach that implies the classical treatment of the electromagnetic field and quantal treatment of the atom. The method is applied to the nonperturbative study of HG by the hydrogen atom in strong laser fields with the fundamental frequencies 532 and $775 \mathrm{~nm}$ and their third harmonics. The results show a strong dependence on the relative phase $\delta$ between the fundamental frequency field and its harmonic. For the intensities used in calculations $\left(1 \times 10^{13}\right.$ and $5 \times 10^{13} \mathrm{~W} / \mathrm{cm}^{2}$ for the fundamental frequency $532 \mathrm{~nm}$ and $1 \times 10^{13}$ and $3 \times 10^{13} \mathrm{~W} / \mathrm{cm}^{2}$ for the fundamental frequency $775 \mathrm{~nm}$, the harmonic intensity being 10 and 100 times weaker), the total photon emission rate has its maximum at $\delta=0$ and minimum at $\delta=\pi$. However, this tendency, while valid for the first several HG peaks, is reversed for the higher HG peaks. The HG spectrum for $\delta=\pi$ is broader and the peak heights decrease more slowly compared to the case of $\delta=0$. These results have their analog in the multiphoton above-threshold detachment study performed recently for $\mathrm{H}^{-}$ions [Telnov, Wang, and Chu, Phys. Rev. A 51, 4797 (1995)].
\end{abstract}

PACS number(s): $32.80 . \mathrm{Rm}, 32.80 . \mathrm{Fb}, 42.50 . \mathrm{Hz}$

\section{INTRODUCTION}

The study of atomic and molecular multiphoton processes in two strong laser fields with different frequencies has received considerable attention both experimentally [1] and theoretically $[2,3]$ in recent years. In the case of atoms, most two-color studies have been confined to the investigation of the phase-dependence effects in multiphoton above-threshold ionization (ATI) [1-3]. It has been found, for example, that the total ionization rate, the electron energy, and the angular distribution depend strongly on the relative intensity and relative phase of the two radiation fields, usually consisting of a fundamental and one of its harmonic fields. In the case of diatomic molecules, it has been predicted, for example, that by tuning the relative phase of the two laser beams, the internuclear potential-energy surface can be modified significantly, suggesting some degree of "coherent control" of the dynamics of multiphoton above-threshold dissociation and chemical bond hardening processes may be feasible [4].

In parallel to the recent extensive studies of the electron emission from multiphoton ATI of atoms, there is also considerable interest in the investigation of photon emission, namely, high-order harmonic generation (HG), that might accompany the excitation or ionization processes. Such high harmonic generation represents a potential practical source of coherent extreme ultraviolet radiation. Most experimental and theoretical studies of $\mathrm{HG}$ processes in strong fields have been mainly confined to the one-color laser field case. (For a

\footnotetext{
*Permanent address: Institute of Physics, St. Petersburg State University, 198904 St. Petersburg, Russia. Electronic address: photon94@kuhub.cc.ukans.edu
}

recent review on high-order harmonic generation in strong fields, see, e.g., Ref. [5] and references therein.) Recently an experiment on the phase-dependent effects on high-order HG by a strong laser field and its third harmonic has been reported [6]. The two-color interference effects on HG were clearly seen. Also very promising are the recent experiments on the generation of short-wavelength radiation by mixed laser fields with commensurate [7] and incommensurate frequencies [8].

In the presence of intense one-color laser fields, several nonperturbative methods have been used in the theoretical studies of HG processes: time-independent Floquet formalism $[9,10]$ and numerical integration of the time-dependent Schrödinger equation [11], as well as purely classical approach to HG [12]. Such theoretical treatments usually consider the interaction of a single atom with strong, classical electromagnetic fields. The investigation of the role of phasematching effects indicates that, under the conditions of low pressure and weak focus typically encountered in experiments, the phase-matched results do not differ significantly from the single-atom results (see, e.g., the review [13]).

In this paper we present a detailed theoretical investigation of the high-order harmonic-generation processes in atomic hydrogen driven by intense two-color laser fields. We found that by varying both the relative phase and the relative intensity of the fundamental to the harmonic field, it is possible to coherently control the enhancement or the decrement of the HG yields. In Sec. II we present a time-independent generalized Floquet formulation for the nonperturbative treatment of high-order harmonic generation in two-color laser fields. The numerical procedure for the solution of the complex quasienergy wave function and the calculation of high-order harmonic generation is presented in Sec. III. In 
Sec. IV we discuss the results of $\mathrm{HG}$ calculations for atomic hydrogen in one- and two-color laser fields for various field parameters. This is followed by a conclusion in Sec. V.

\section{THEORY}

We will follow the theory of harmonic generation that treats the atom quantum mechanically and the radiation classically. This approach is well justified in most of cases from the point of view of the theory that treats the radiation field quantum mechanically [14]. According to the classical theory of fields [15], the intensity of radiation produced by an accelerated charge per unit solid angle and summed over all possible polarizations is given by the expression

$$
\frac{d I}{d \Omega}=\frac{|\mathbf{a}(t)|^{2}}{4 \pi c^{3}} \sin ^{2} \vartheta
$$

where $\mathbf{a}(t)$ is the acceleration of the charge, $c$ is the velocity of the light, and $\vartheta$ is the angle (with respect to a) under which the radiation is detected. In the case of periodic motion, the acceleration $\mathbf{a}(t)$ can be expanded in Fourier series

$$
\mathbf{a}(t)=\sum_{n=-\infty}^{\infty} \mathbf{a}_{n} \exp (-i n \omega t)
$$

where the frequency $\omega=2 \pi / T, T$ being the period. The total (angle-integrated) intensity of the $n$th harmonic is

$$
I_{n}=\frac{4\left|\mathbf{a}_{n}\right|^{2}}{3 c^{3}} \text {. }
$$

Since the acceleration Fourier components $\mathbf{a}_{n}$ and displacement Fourier components $\mathbf{d}_{n}$ are related by

$$
\mathbf{a}_{n}=-n^{2} \omega^{2} \mathbf{d}_{n},
$$

the intensity of the $n$th harmonic can be written also in the length form

$$
I_{n}=\frac{4 n^{4} \omega^{4}\left|\mathbf{d}_{n}\right|^{2}}{3 c^{3}}
$$

In our case the charge particle is represented by an electron moving under the influence of the core and external laser field. Let the atomic potential $W(r)$ be spherically symmetrical and the external field linearly polarized in the $z$ direction. Then the Hamiltonian $\hat{H}$ reads (in a.u.)

$$
\hat{H}=-\frac{1}{2} \nabla^{2}+W(r)+z F(t),
$$

where $F(t)$ is the laser field strength. For the two-color fields one has the expression for $F(t)$,

$$
F(t)=F_{1} \cos \left(\omega_{1} t\right)+F_{2} \cos \left(\omega_{2} t+\delta\right),
$$

with $F_{1}$ and $F_{2}$ being the field strengths for the first and the second fields, respectively, and $\delta$ being the relative phase shift between the two fields. ( $\delta$ is meaningful for commensurate frequencies $\omega_{1}$ and $\omega_{2}$ only; see the discussion below.) In the present formulation, the atomic system will be treated quantum mechanically. Thus the acceleration $\mathbf{a}(t)$ and its Fourier components should be replaced with the corresponding quantum expectation values. (For simplicity, we shall keep the same notation for the quantum expectation values as for the corresponding classical values.) If the initial state of the atom is spherically symmetrical, then only the $z$ component of the mean acceleration does not vanish. According to the Ehrenfest theorem [16],

$$
\frac{d^{2}}{d t^{2}}\langle z\rangle=\frac{i}{m}\left\langle\Psi\left|\left[\hat{H}, \hat{p}_{z}\right]\right| \Psi\right\rangle
$$

where we assume that $\Psi(\mathbf{r}, t)$ is a quasienergy wave function describing the electron subject to the influence of the core as well as the external field, $\hat{p}_{z}$ being the momentum operator.

Consider first the case of commensurate frequencies $\omega_{1}$ and $\omega_{2}$. Here the ordinary Floquet theory applies and the wave function $\Psi(\mathbf{r}, t)$ can be expanded in the Fourier series with a single fundamental frequency $\omega$ ( $\varepsilon$ is a quasienergy):

$$
\Psi(\mathbf{r}, t)=\exp (-i \varepsilon t) \sum_{m=-\infty}^{\infty} \psi_{m}(\mathbf{r}) \exp (-i m \omega t)
$$

If the second laser field is a harmonic of the first one

$$
\omega_{2}=N \omega_{1}
$$

where $N$ is an integer, the fundamental frequency $\omega$ is equal to the frequency of the first field

$$
\omega=\omega_{1},
$$

and the expression for the squared absolute value of the Fourier component $\left|\mathbf{a}_{n}\right|^{2}$ can be written as

$$
\begin{aligned}
\left|\mathbf{a}_{n}\right|^{2}= & \mid \sum_{m=-\infty}^{\infty}\left\langle\psi_{m-n}\left|\frac{z}{r} \frac{d W}{d r}\right| \psi_{m}\right\rangle+\frac{1}{2} F_{1}\left[\delta_{n, 1}+\delta_{n,-1}\right] \\
& +\left.\frac{1}{2} F_{2}\left[\delta_{n, N} \exp (-i \delta)+\delta_{n-N} \exp (i \delta)\right]\right|^{2}
\end{aligned}
$$

Here we have evaluated the commutator $\left[\hat{H}, \hat{p}_{z}\right] ; \delta_{n k}$ is a Kronecker symbol and $\delta$ without subscripts is a relative phase between the two laser fields. In analogy, the squared absolute value of the Fourier component $\left|\mathbf{d}_{n}\right|^{2}$ is expressed as

$$
\left|\mathbf{d}_{n}\right|^{2}=\left|\sum_{m=-\infty}^{\infty}\left\langle\psi_{m-n}|z| \psi_{m}\right\rangle\right|^{2}
$$

Let the integer number $N$, which describes the second (harmonic) field, be an odd number. Then, for the spherically symmetrical atomic potential and the initial state with definite parity, the Fourier components of the wave function $\Psi(\mathbf{r}, t)$ also possess definite parity, which is different for even and odd components. As one can see from Eq. (12), only the odd Fourier components are present in the mean acceleration due to parity restrictions. 
The value $I_{n}$ given by Eqs. (3) and (5) has the meaning of the energy radiated per unit time through the mode with the frequency $n \omega$. The corresponding photon emission rate $\Gamma_{n}$ (the number of photons of frequency $n \omega$ emitted per unit time) is obtained from (3) and (5) by dividing by $n \omega$ :

$$
\Gamma_{n}=\frac{4\left|\mathbf{a}_{n}\right|^{2}}{3 n \omega c^{3}}=\frac{4 n^{3} \omega^{3}\left|\mathbf{d}_{n}\right|^{2}}{3 c^{3}} .
$$

Both forms (acceleration and length) are equivalent in the Floquet theory; in practice, one needs to take care of the proper "regularization" of the integral (13), which is formally divergent. Strictly speaking, the acceleration form integral (8) is also formally divergent due to the imaginary part of the quasienergy (resonance state). However, for the intensities used in the calculations, the imaginary part of the quasienergy is negligibly small, so no special regularization is required for numerical calculation of the integral (8). For higher intensities one may need to use the regularization for the acceleration form integral as well as for the length form integral (see Sec. III).

Now consider briefly the general case of incommensurate frequencies $\omega_{1}$ and $\omega_{2}$. According to the many-mode Floquet theory $[9,17]$, the wave function $\Psi(\mathbf{r}, t)$ can be expanded in a double Fourier series with two fundamental frequencies $\omega_{1}$ and $\omega_{2}$ :

$$
\begin{aligned}
\Psi(\mathbf{r}, t)= & \exp (-i \varepsilon t) \sum_{m_{1}, m_{2}} \psi_{m_{1} m_{2}}(\mathbf{r}) \\
& \times \exp \left[-i\left(m_{1} \omega_{1}+m_{2} \omega_{2}\right) t\right]
\end{aligned}
$$

The long-time average of the squared acceleration is equal to

$$
\lim _{T \rightarrow \infty} \frac{1}{T} \int_{0}^{T}|\mathbf{a}(t)|^{2} d t=\sum_{n_{1}, n_{2}=-\infty}^{\infty}\left|\mathbf{a}_{n_{1} n_{2}}\right|^{2}
$$

with the squared absolute value of the Fourier components $\left|\mathbf{a}_{n_{1} n_{2}}\right|^{2}$ defined as

$$
\begin{aligned}
\left|\mathbf{a}_{n_{1} n_{2}}\right|^{2}= & \mid \sum_{m_{1}, m_{2}}\left\langle\psi_{m_{1}-n_{1}, m_{2}-n_{2}}\left|\frac{z}{r} \frac{d W}{d r}\right| \psi_{m_{1} m_{2}}\right\rangle \\
& +\frac{1}{2} F_{1}\left(\delta_{n_{1}, 1}+\delta_{n_{1},-1}\right) \delta_{n_{2}, 0} \\
& +\left.\frac{1}{2} F_{2}\left[\delta_{n_{2}, 1} \exp (-i \delta)+\delta_{n_{2},-1} \exp (i \delta)\right] \delta_{n_{1}, 0}\right|^{2} .
\end{aligned}
$$

Note that, although the phase shift $\delta$ is present in Eq. (17), it does not affect the result. The Fourier components of the wave function $\psi_{m_{1} m_{2}}$ depend on $\delta$ via the factor exp $\left(-i m_{2} \delta\right.$ ) only (see the discussion in Ref. [3]), so the squared absolute value of the Fourier components of the acceleration do not depend on it. When driven in the two-color fields with incommensurate frequencies $\omega_{1}$ and $\omega_{2}$, the electron can emit radiation with the frequencies $\left|n_{1} \omega_{1}+n_{2} \omega_{2}\right|$, where $n_{1}$ and $n_{2}$ are integers. The corresponding photon emission rate is given by

$$
\Gamma_{n_{1} n_{2}}=\frac{4\left|\mathbf{a}_{n_{1} n_{2}}\right|^{2}}{3\left|n_{1} \omega_{1}+n_{2} \omega_{2}\right| c^{3}}
$$

\section{NUMERICAL PROCEDURES}

In this paper, we consider the high-order harmonic generation by the hydrogen atom driven by the fundamental frequency laser field and its third harmonic

$$
F(t)=F_{1} \cos (\omega t)+F_{2} \cos (3 \omega t+\delta)
$$

When both external fields are polarized in the same direction, the projection of the electron angular momentum onto the field axis is an integral of motion. Since the ground state of the hydrogen atom is under consideration, this projection is equal to zero. Thus the quasienergy wave function $\Psi(\mathbf{r}, t)$ can be expanded in the double series over the time Fourier components and Legendre polynomials $P_{l}(\cos \vartheta)$, $\vartheta$ being the angle between $\mathbf{r}$ and the field direction ( $z$ axis):

$$
\begin{aligned}
\Psi(\mathbf{r}, t)= & \exp (-i \varepsilon t) \sum_{m=-\infty}^{\infty} \exp (-i m \omega t) \\
& \times \sum_{l=0}^{\infty}\left(\frac{l+1}{2}\right)^{1 / 2} \frac{1}{r} \psi_{m l}(r) P_{l}(\cos \vartheta)
\end{aligned}
$$

In the presence of strong fields, the quasienergy eigenstate corresponding to the ground state is a metastable resonance state with complex quasienergy $\varepsilon=\left(E_{R},-\Gamma / 2\right)$. The real part of the quasienergy $\left(E_{R}\right)$ provides the ac Stark shift of the ground state whereas the imaginary part $(\Gamma)$ gives rise to the total multiphoton ionization rate. This quasienergy resonance state, corresponding to a complex role in the higher Riemann sheet, can be determined by means of the nonHermitian Floquet Hamiltonian formalism [9]. In this formalism, we first substitute Eq. (20) into the time-dependent Schrödinger equation, yielding a time-independent Floquet Hamiltonian $\hat{H}_{F}(\mathbf{r})$. We then analytically continue $\hat{H}_{F}(\mathbf{r})$ into the complex plane on higher Riemann sheet by means of the complex scaling transformation [18], $\mathbf{r} \rightarrow \mathbf{r} \exp (i \alpha)$. The solution of the non-Hermitian Floquet Hamiltonian matrix $\hat{H}_{F}[\mathbf{r} \exp (i \alpha)] \equiv \hat{H}_{F}(\alpha)$ so obtained provides directly the desirable complex quasienergies $\varepsilon$ quasienergy eigenfunctions $\Psi_{\varepsilon}(\mathbf{r}, t)$ sought. In this paper we use the complexscaling generalized pseudospectral technique [19] recently developed for the discretization and solution of the nonHermitian Floquet Hamiltonian $\hat{H}_{F}(\alpha)$. The computational efficiency and advantages of this discretization method over the traditional basis-set-expansion variational method have been demonstrated elsewhere [19]. To facilitate the conver- 
gency in strong-field calculations, we use the velocity-gauge Hamiltonian instead of the length-gauge one provided by Eq. (6):

$$
\hat{H}=-\frac{1}{2} \nabla^{2}+W(r)-i A(t) \frac{d}{d z},
$$

$$
F(t)=-\frac{d}{d t} A(t)
$$

The set of coupled equations for the components $\psi_{m l}(r)$, from Eq. (20), now takes the form

$$
\begin{aligned}
& {\left[\left(-\frac{1}{2} \frac{d^{2}}{d r^{2}}+\frac{l(l+1)}{2 r^{2}}\right) e^{-i 2 \alpha}+W\left(r e^{i \alpha}\right)-\varepsilon-m \omega\right] \psi_{m l}+\frac{F_{1}}{2 \omega} e^{-i \alpha}\left[\frac{l}{\sqrt{(2 l-1)(2 l+1)}}\left(-\frac{d}{d r}+\frac{l}{r}\right) \psi_{(m-1),(l-1)}\right.} \\
& +\frac{l+1}{\sqrt{(2 l+1)(2 l+3)}}\left(-\frac{d}{d r}-\frac{l+1}{r}\right) \psi_{(m-1),(l+1)}+\frac{l}{\sqrt{(2 l-1)(2 l+1)}}\left(\frac{d}{d r}-\frac{l}{r}\right) \psi_{(m+1),(l-1)} \\
& \left.+\frac{l+1}{\sqrt{(2 l+1)(2 l+3)}}\left(\frac{d}{d r}+\frac{l+1}{r}\right) \psi_{(m+1),(l+1)}\right]+\frac{F_{2}}{2 N \omega} e^{-i \alpha}\left[\frac{l}{\sqrt{(2 l-1)(2 l+1)}}\left(-\frac{d}{d r}+\frac{l}{r}\right) e^{-i \delta} \psi_{(m-N),(l-1)}\right. \\
& +\frac{l+1}{\sqrt{(2 l+1)(2 l+3)}}\left(-\frac{d}{d r}-\frac{l+1}{r}\right) e^{-i \delta} \psi_{(m-N),(l+1)}+\frac{l}{\sqrt{(2 l-1)(2 l+1)}}\left(\frac{d}{d r}-\frac{l}{r}\right) e^{i \delta} \psi_{(m+N),(l-1)} \\
& \left.+\frac{l+1}{\sqrt{(2 l+1)(2 l+3)}}\left(\frac{d}{d r}+\frac{l+1}{r}\right) e^{i \delta} \psi_{(m+N),(l+1)}\right]=0 .
\end{aligned}
$$

Then we apply the mapping transformation to the variable $r$ $([0, \infty] \rightarrow[-1,1])$ and discretize it according to the complex-scaling generalized pseudospectral method [19]. Depending upon the relative phase $\delta$, the resulting discretized Floquet Hamiltonian matrix $\hat{H}_{F}(\alpha)$ can be complex symmetric or asymmetric.

For high-order harmonic-generation calculation, highquality quasienergy wave functions are required to achieve convergency. The size of the non-Hermitian Floquet matrix depends upon the laser frequency and intensity used. Typically we used 40-50 mesh points for the discretization of the radial coordinate. Sufficient numbers of partial waves and Floquet photon blocks are included to achieve convergency to desirable accuracy. The Floquet matrix so constructed is a large sparse complex matrix, typically of the order of 10000 $\times 10000$ or more. We extended the implicitly restarted Arnoldi algorithm [20] with spectral transformation to facilitate the solution of the selected eigenvalues and eigenfunctions. By further taking into account the sparse structure in the Floquet Hamiltonian, we have achieved a computationally stable and efficient procedure for the solution of the internal spectrum of complex sparse matrix of large dimensions. As an example, for a complex matrix size of the order of 15000 $\times 15000$, it takes about a total of $30 \mathrm{~s}$ in CRAY-YMP for the solution of selective eigenvalues and eigenfunctions. The Arnoldi algorithm has the additional advantage that it does not suffer the problem of generating undesirable repeated roots as often encountered in the Lanczos algorithm. The detailed discussion of the numerical procedure will be presented elsewhere.

The integrations over the angles $\vartheta$ and $\phi$ (in the spherical coordinate system with the polar axis along the field direc- tion) with the function (20) in Eqs. (8) and (13) can be performed analytically giving the following expressions for the squared Fourier components of the acceleration and displacement, respectively:

$$
\begin{aligned}
\left|\mathbf{a}_{n}\right|^{2}= & -\sum_{m, l} \frac{l+1}{\sqrt{(2 l+1)(2 l+3)}}\left[\left\langle\psi_{(m-n), l}\left|\frac{1}{r^{2}}\right| \psi_{m,(l+1)}\right\rangle\right. \\
& \left.+\left\langle\psi_{(m-n),(l+1)}\left|\frac{1}{r^{2}}\right| \psi_{m, l}\right\rangle\right]+\frac{1}{2} F_{1} \delta_{n, 1}+\frac{1}{2} F_{1} \delta_{n,-1} \\
& +\frac{1}{2} F_{2} \exp (-i \delta) \delta_{n, 3}+\left.\frac{1}{2} F_{2} \exp (i \delta) \delta_{n,-3}\right|^{2} \\
\left|\mathbf{d}_{n}\right|^{2}= & \mid \sum_{m, l} \frac{l+1}{\sqrt{(2 l+1)(2 l+3)}}\left[\left\langle\psi_{(m-n), l}|r| \psi_{m,(l+1)}\right\rangle\right. \\
& \left.+\left\langle\psi_{(m-n),(l+1)}|r| \psi_{m, l}\right\rangle\right]\left.\right|^{2}
\end{aligned}
$$

The matrix elements in Eqs. (24) and (25) involve the integrations with respect to the radial variable $r$ only. Note that the wave functions obtained from Eq. (23) are given for the complex $r$ values (rotated by the angle $\alpha$ in the complex $r$ plane), while the Eqs. (24) and (25) imply that the wave functions are taken for real $r$.

As mentioned above, the acceleration and length forms of the expression for HG rates are equivalent in the Floquet 
TABLE I. Harmonic-generation rates by the hydrogen atom for the fundamental field of frequency $532 \mathrm{~nm}$ calculated with acceleration and length forms. $A$, acceleration form calculated with the complex rotated-wave functions; $B$, length form calculated with the complex rotated-wave functions. The number in brackets indicates the power of 10 .

\begin{tabular}{lcccc}
\hline \hline & \multicolumn{4}{c}{ Harmonic-generation rates (a.u.) } \\
\cline { 2 - 5 }$n$ & $1 \times 10^{13} \mathrm{~W} / \mathrm{cm}^{2}$ & $5 \times 10^{13} \mathrm{~W} / \mathrm{cm}^{2}$ \\
\hline 3 & $A$ & $B$ & $A$ & $B$ \\
5 & $2.43[-15]$ & $2.43[-15]$ & $2.18[-13]$ & $2.18[-13]$ \\
7 & $1.60[-16]$ & $1.60[-16]$ & $1.12[-13]$ & $1.12[-13]$ \\
9 & $1.96[-19]$ & $1.96[-19]$ & $7.28[-15]$ & $7.28[-15]$ \\
11 & $5.49[-23]$ & $5.49[-23]$ & $3.03[-16]$ & $3.03[-16]$ \\
13 & $6.02[-27]$ & $6.02[-27]$ & $3.07[-18]$ & $3.07[-18]$ \\
15 & $3.37[-31]$ & $3.37[-31]$ & $1.10[-20]$ & $1.10[-20]$ \\
17 & $1.09[-35]$ & $1.08[-35]$ & $1.87[-23]$ & $1.87[-23]$ \\
19 & & & $1.81[-26]$ & $1.81[-26]$ \\
21 & & & $1.10[-29]$ & $1.10[-29]$ \\
23 & & & $4.62[-33]$ & $4.70[-33]$ \\
\hline \hline
\end{tabular}

theory, if an appropriate regularization of the length form integral is performed. The integration in the complex coordinate $r$ plane can be considered as such a regularization since the complex rotated-wave functions decrease exponentially as $r \rightarrow \infty$ and ensure the convergence of the integral (25). In this case, due to biorthogonality of the complex scaled wave functions [18], the complex conjugation should not be applied to any radial function in Eq. (25). Here we first present the results of $\mathrm{HG}$ calculations in one-color laser field using acceleration and length forms (Table I). The excellent agreement of the acceleration and length forms shows the self-consistence of the approach and the quality of the quasienergy wave functions. However, the acceleration form is generally expected to be more stable and reliable [11(b),21], especially for high-order harmonics, because it makes use of the coordinate $r$ range, which is not far from the nucleus, where the wave functions calculated are more accurate. That is why we used the acceleration form of the
HG rate expression to obtain the results for two-color fields presented in the next section.

\section{RESULTS}

The calculations of HG rates were performed for the fundamental frequencies $\omega=8.564539 \times 10^{-2}$ a.u. (corresponding to the wavelength $532 \mathrm{~nm}$ ) and $\omega=5.879142 \times 10^{-2}$ a.u. (corresponding to the wavelength $775 \mathrm{~nm}$ ). The third harmonic was used for the two-color calculations. The fundamental frequency field intensities $I_{L}$ used are $10^{13}$ and $5 \times 10^{13} \mathrm{~W} / \mathrm{cm}^{2}$ for the wavelength $532 \mathrm{~nm}$ and $10^{13}$ and $3 \times 10^{13} \mathrm{~W} / \mathrm{cm}^{2}$ for the wavelength $775 \mathrm{~nm}$. The thirdharmonic field intensity $I_{H}$ was chosen to be 10 or 100 times weaker than that of the fundamental frequency field and the relative phases are $\delta=0$ and $\pi$. The results are presented in Tables II-V and in Figs. 1-4.

For the fundamental wavelength $532 \mathrm{~nm}$ (Tables II and III and Figs. 1 and 2), one can see that for rather strong harmonic field (10 times weaker than the fundamental one) the HG rates are generally significantly enhanced, compared with the one-color case, for both the phase $\delta$ values used in the calculations. However, for the weaker harmonic intensity (100 times weaker than the fundamental field) and $\delta=\pi$, the rates for the first few harmonics are smaller than that for the one-color HG. We see that a small admixture of the harmonic field can lead to a dramatic change in the HG rates. Thus, by tuning the relative phase and the relative intensity of the fundamental to the harmonic field, one can control the enhancement or decrement of the HG yield. The dependence of the HG rates on the relative phase $\delta$ is the same for both strong and weak harmonic fields, as well as for both fundamental field intensities. For the first few generated harmonics (the exact number depends on the intensities; see Tables I and II and Figs. 1 and 2) the largest rate is observed for $\delta=0$ and the smallest for $\delta=\pi$. Then the tendency is inverted and the highest calculated HG rates $\left(15-17\right.$ for $10^{13}$ $\mathrm{W} / \mathrm{cm}^{2}$ and $15-23$ for $5 \times 10^{13} \mathrm{~W} / \mathrm{cm}^{2}$ ) show the dominance of the $\delta=\pi$ results over $\delta=0$. The exception to this rule is made by the seventh harmonic for the fundamental field intensity $I_{L}=5 \times 10^{13} \mathrm{~W} / \mathrm{cm}^{2}$ where the rate for $\delta=\pi$ ex-

TABLE II. Harmonic-generation rates by the hydrogen atom for the fundamental field of frequency $532 \mathrm{~nm}$, intensity $1 \times 10^{13} \mathrm{~W} / \mathrm{cm}^{2}$, and its third harmonic; $n$ is the harmonic order. The number in brackets indicates the power of 10 .

\begin{tabular}{|c|c|c|c|c|c|}
\hline \multirow[b]{4}{*}{$n$} & \multicolumn{5}{|c|}{ Harmonic generation rates (a.u.) } \\
\hline & \multirow{3}{*}{$\begin{array}{l}\text { One-color } \\
\text { fundamental } \\
\text { field }\end{array}$} & \multicolumn{4}{|c|}{ Two-color harmonic field } \\
\hline & & \multicolumn{2}{|c|}{$1 \times 10^{11} \mathrm{~W} / \mathrm{cm}^{2}$} & \multicolumn{2}{|c|}{$1 \times 10^{9} \mathrm{~W} / \mathrm{cm}^{2}$} \\
\hline & & $\delta=0$ & $\delta=\pi$ & $\delta=0$ & $\delta=\pi$ \\
\hline 3 & $2.43[-15]$ & $5.60[-13]$ & $4.14[-13]$ & $1.41[-14]$ & $4.24[-16]$ \\
\hline 5 & $5.78[-15]$ & $3.82[-13]$ & $2.36[-13]$ & $1.72[-14]$ & $4.35[-16]$ \\
\hline 7 & $1.60[-16]$ & $5.11[-15]$ & $1.37[-15]$ & $3.25[-16]$ & $5.61[-17]$ \\
\hline 9 & $1.96[-19]$ & $8.20[-18]$ & $2.94[-18]$ & $3.50[-19]$ & $8.85[-20]$ \\
\hline 11 & $5.49[-23]$ & $1.29[-20]$ & $3.96[-21]$ & $8.39[-23]$ & $3.41[-23]$ \\
\hline 13 & $6.02[-27]$ & $9.58[-24]$ & $5.23[-24]$ & $8.27[-27]$ & $6.87[-27]$ \\
\hline 15 & $3.37[-31]$ & $6.96[-27]$ & $3.85[-27]$ & $5.48[-31]$ & $9.13[-31]$ \\
\hline 17 & $1.09[-35]$ & $3.82[-30]$ & $3.84[-30]$ & $2.83[-35]$ & $7.93[-35]$ \\
\hline
\end{tabular}


TABLE III. Harmonic-generation rates by the hydrogen atom for the fundamental field of frequency $532 \mathrm{~nm}$, intensity $5 \times 10^{13} \mathrm{~W} / \mathrm{cm}^{2}$, and its third harmonic; $n$ is the harmonic order. The number in brackets indicates the power of 10 .

\begin{tabular}{|c|c|c|c|c|c|}
\hline \multirow[b]{4}{*}{$n$} & \multicolumn{5}{|c|}{ Harmonic-generation rates (a.u.) } \\
\hline & \multirow{3}{*}{$\begin{array}{l}\text { One-color } \\
\text { fundamental } \\
\text { field }\end{array}$} & \multicolumn{4}{|c|}{ Two-color harmonic field } \\
\hline & & \multicolumn{2}{|c|}{$5 \times 10^{11} \mathrm{~W} / \mathrm{cm}^{2}$} & \multicolumn{2}{|c|}{$5 \times 10^{9} \mathrm{~W} / \mathrm{cm}^{2}$} \\
\hline & & $\delta=0$ & $\delta=\pi$ & $\delta=0$ & $\delta=\pi$ \\
\hline 3 & $2.18[-13]$ & $5.14[-12]$ & $1.77[-12]$ & $4.19[-13]$ & $8.20[-14]$ \\
\hline 5 & $1.64[-12]$ & $4.54[-12]$ & $2.43[-12]$ & $1.75[-12]$ & $1.57[-12]$ \\
\hline 7 & $1.12[-13]$ & $5.90[-13]$ & $1.71[-12]$ & $9.07[-14]$ & $1.55[-13]$ \\
\hline 9 & $7.28[-15]$ & $1.19[-13]$ & $8.45[-14]$ & $1.02[-14]$ & $6.25[-15]$ \\
\hline 11 & $3.03[-16]$ & $2.68[-15]$ & $1.41[-15]$ & $4.17[-16]$ & $2.20[-16]$ \\
\hline 13 & $3.07[-18]$ & $1.45[-17]$ & $1.35[-17]$ & $3.68[-18]$ & $2.51[-18]$ \\
\hline 15 & $1.10[-20]$ & $6.72[-20]$ & $8.49[-20]$ & $1.10[-20]$ & $1.07[-20]$ \\
\hline 17 & $1.87[-23]$ & $2.03[-22]$ & $3.94[-22]$ & $1.45[-23]$ & $2.34[-23]$ \\
\hline 19 & $1.81[-26]$ & $4.98[-25]$ & $1.46[-24]$ & $9.94[-27]$ & $3.13[-26]$ \\
\hline 21 & $1.10[-29]$ & $9.89[-28]$ & $4.50[-27]$ & $3.82[-30]$ & $2.86[-29]$ \\
\hline 23 & $4.62[-33]$ & $1.67[-30]$ & $1.20[-29]$ & $8.35[-34]$ & $1.94[-32]$ \\
\hline
\end{tabular}

ceeds that for $\delta=0$. This result can be attributed to some resonance enhancement of $\mathrm{HG}$, which takes place only for the relative phase $\delta=\pi$. The general observation is that the HG spectrum is broader for $\delta=\pi$ than for $\delta=0$ and the rates decrease more slowly in the tail portion of the spectrum, whereas in the top portion (the first few harmonics) they have the magnitudes smaller than that for $\delta=0$. These results are analogous to our previous results for above-threshold detachment by two-color laser fields from $\mathrm{H}^{-}$negative ions [3].

For the fundamental wavelength $775 \mathrm{~nm}$ (Tables IV and V and Figs. 3 and 4), the features observed for the wavelength $532 \mathrm{~nm}$ are confirmed. Since the wavelength is longer, the plateau region, where the harmonic-generation rates are comparable in magnitude, is broader than that for $532 \mathrm{~nm}$ (third to thirteenth harmonic for the intensity $I_{L}=3 \times 10^{13}$ $\mathrm{W} / \mathrm{cm}^{2}$ ). In this region the rates exhibit irregular oscillatory dependence on the harmonic order, more pronounced for the stronger fundamental field $\left(I_{L}=3 \times 10^{13} \mathrm{~W} / \mathrm{cm}^{2}\right)$. For example, the rates of the harmonics from the ninth to the fifteenth for the third-harmonic field intensity $I_{H}=3 \times 10^{11}$ $\mathrm{W} / \mathrm{cm}^{2}$ and the relative phase $\delta=\pi$ are smaller than that for the one-color $\mathrm{HG}$ and the third-harmonic field intensity $I_{H}=3 \times 10^{9} \mathrm{~W} / \mathrm{cm}^{2}$. Such a behavior is not observed for the weaker fundamental frequency field $\left(I_{L}=10^{13} \mathrm{~W} / \mathrm{cm}^{2}\right)$ or for the shorter wavelength $(532 \mathrm{~nm})$ at any intensity used in the calculations. One can see also that the difference in HG rates for different relative phases $(0$ and $\pi)$ at the same intensities of the fundamental and harmonic fields is larger for the weaker fundamental frequency field $\left(I_{L}=10^{13} \mathrm{~W} / \mathrm{cm}^{2}\right)$. The same tendency is observed for the wavelength $532 \mathrm{~nm}$.

The dependence of $\mathrm{HG}$ rates on the relative phase described above can be understood to some extent with the help of the simple semiclassical theory [22]. According to this theory, HG is produced by the electrons excited into the

TABLE IV. Harmonic-generation rates by the hydrogen atom for the fundamental field of frequency $775 \mathrm{~nm}$, intensity $1 \times 10^{13} \mathrm{~W} / \mathrm{cm}^{2}$, and its third harmonic; $n$ is the harmonic order. The numbe in brackets indicates the power of 10 .

\begin{tabular}{|c|c|c|c|c|c|}
\hline \multirow[b]{4}{*}{$n$} & \multicolumn{5}{|c|}{ Harmonic-generation rates (a.u.) } \\
\hline & \multirow{3}{*}{$\begin{array}{l}\text { One-color } \\
\text { fundamental } \\
\text { field }\end{array}$} & \multicolumn{4}{|c|}{ Two-color harmonic field } \\
\hline & & \multicolumn{2}{|c|}{$1 \times 10^{11} \mathrm{~W} / \mathrm{cm}^{2}$} & \multicolumn{2}{|c|}{$1 \times 10^{9} \mathrm{~W} / \mathrm{cm}^{2}$} \\
\hline & & $\delta=0$ & $\delta=\pi$ & $\delta=0$ & $\delta=\pi$ \\
\hline 3 & $1.51[-16]$ & $7.52[-14]$ & $6.16[-14]$ & $1.48[-15]$ & $1.90[-16]$ \\
\hline 5 & $1.88[-17]$ & $3.79[-15]$ & $1.34[-15]$ & $8.65[-17]$ & $6.05[-19]$ \\
\hline 7 & $8.49[-17]$ & $1.37[-14]$ & $1.04[-15]$ & $2.90[-16]$ & $4.41[-18]$ \\
\hline 9 & $3.84[-17]$ & $3.72[-15]$ & $6.16[-16]$ & $1.11[-16]$ & $4.46[-18]$ \\
\hline 11 & $2.14[-18]$ & $1.33[-16]$ & $1.52[-17]$ & $4.93[-18]$ & $5.75[-19]$ \\
\hline 13 & $1.96[-20]$ & $9.97[-19]$ & $1.47[-19]$ & $3.98[-20]$ & $7.07[-21]$ \\
\hline 15 & $5.08[-23]$ & $3.78[-21]$ & $1.08[-21]$ & $8.88[-23]$ & $2.30[-23]$ \\
\hline 17 & $5.52[-26]$ & $1.02[-23]$ & $4.56[-24]$ & $7.80[-26]$ & $3.31[-26]$ \\
\hline 19 & $3.13[-29]$ & $1.97[-26]$ & $1.43[-26]$ & $3.35[-29]$ & $2.73[-29]$ \\
\hline 21 & $1.05[-32]$ & $3.10[-29]$ & $3.45[-29]$ & $8.33[-33]$ & $1.48[-32]$ \\
\hline 23 & $2.27[-36]$ & $3.99[-32]$ & $6.72[-32]$ & $1.36[-36]$ & $5.68[-36]$ \\
\hline
\end{tabular}


TABLE V. Harmonic-generation rates by the hydrogen atom for the fundamental field of frequency $775 \mathrm{~nm}$, intensity $3 \times 10^{13} \mathrm{~W} / \mathrm{cm}^{2}$, and its third harmonic; $n$ is the harmonic order. The number in brackets indicates the power of 10 .

\begin{tabular}{|c|c|c|c|c|c|}
\hline \multirow[b]{4}{*}{$n$} & \multicolumn{5}{|c|}{ Harmonic-generation rates (a.u.) } \\
\hline & \multirow{3}{*}{$\begin{array}{l}\text { One-color } \\
\text { fundamental } \\
\text { field }\end{array}$} & \multicolumn{4}{|c|}{ Two-color harmonic field } \\
\hline & & \multicolumn{2}{|c|}{$3 \times 10^{11} \mathrm{~W} / \mathrm{cm}^{2}$} & \multicolumn{2}{|c|}{$3 \times 10^{9} \mathrm{~W} / \mathrm{cm}^{2}$} \\
\hline & & $\delta=0$ & $\delta=\pi$ & $\delta=0$ & $\delta=\pi$ \\
\hline 3 & $7.78[-15]$ & $3.77[-13]$ & $1.87[-13]$ & $1.97[-14]$ & $1.35[-15]$ \\
\hline 5 & $1.40[-14]$ & $3.39[-13]$ & $7.53[-14]$ & $2.62[-14]$ & $5.88[-15]$ \\
\hline 7 & $2.81[-15]$ & $3.02[-13]$ & $7.21[-14]$ & $8.87[-15]$ & $5.50[-16]$ \\
\hline 9 & $1.22[-14]$ & $4.79[-14]$ & $1.74[-15]$ & $1.45[-14]$ & $1.02[-14]$ \\
\hline 11 & $2.08[-14]$ & $1.50[-13]$ & $7.27[-15]$ & $2.81[-14]$ & $1.46[-14]$ \\
\hline 13 & $3.03[-15]$ & $8.79[-15]$ & $1.59[-16]$ & $3.48[-15]$ & $2.60[-15]$ \\
\hline 15 & $8.38[-17]$ & $2.22[-16]$ & $5.22[-17]$ & $8.70[-17]$ & $8.15[-17]$ \\
\hline 17 & $9.69[-19]$ & $5.21[-18]$ & $2.35[-18]$ & $9.96[-19]$ & $9.99[-19]$ \\
\hline 19 & $6.37[-21]$ & $5.60[-20]$ & $4.92[-20]$ & $6.95[-21]$ & $6.55[-21]$ \\
\hline 21 & $2.75[-23]$ & $4.16[-22]$ & $6.41[-22]$ & $3.13[-23]$ & $2.75[-23]$ \\
\hline 23 & $8.30[-26]$ & $2.27[-24]$ & $5.92[-24]$ & $9.10[-26]$ & $8.31[-26]$ \\
\hline 25 & $1.81[-28]$ & $9.74[-27]$ & $4.23[-26]$ & $1.75[-28]$ & $1.94[-28]$ \\
\hline
\end{tabular}

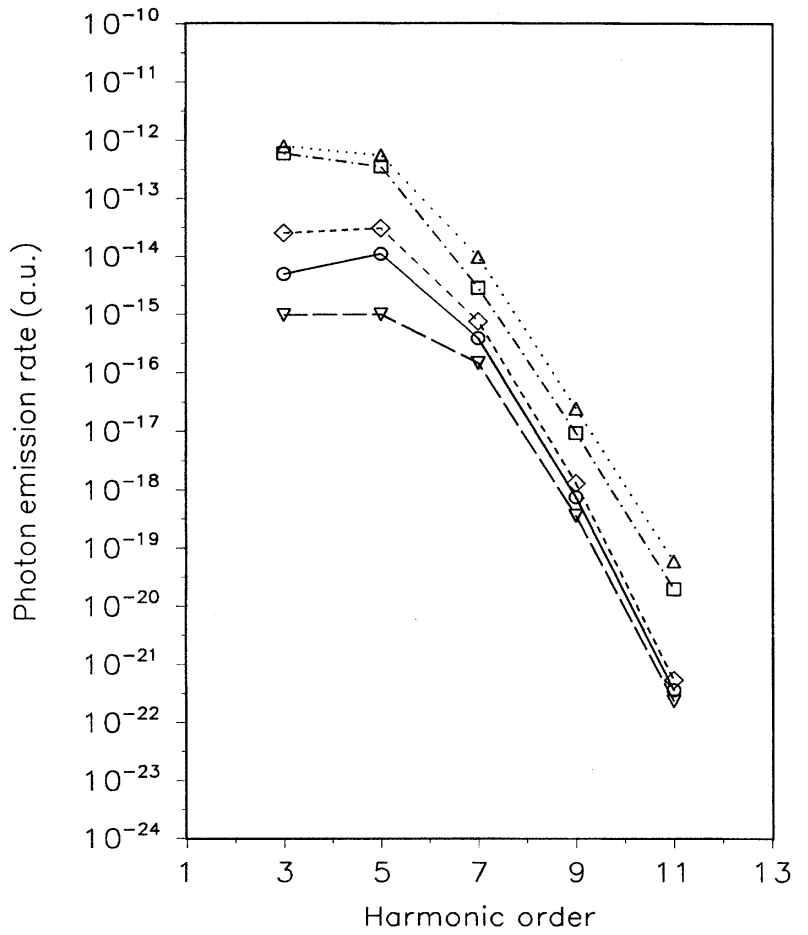

FIG. 1. Harmonic-generation rates (in a.u.) for the fundamental frequency field $532 \mathrm{~nm}$, intensity $1 \times 10^{13} \mathrm{~W} / \mathrm{cm}^{2}$, and its third harmonic. $\bigcirc$, one-color results (fundamental frequency field only); $\diamond$, harmonic field $1 \times 10^{9} \mathrm{~W} / \mathrm{cm}^{2}$, relative phase $\delta=0 ; \nabla$, harmonic field $1 \times 10^{9} \mathrm{~W} / \mathrm{cm}^{2}$, relative phase $\delta=\pi ; \triangle$, harmonic field $1 \times 10^{9} \mathrm{~W} / \mathrm{cm}^{2}$, relative phase $\delta=\pi ; \triangle$, harmonic field $1 \times 10^{11}$ $\mathrm{W} / \mathrm{cm}^{2}$, relative phase $\delta=0 ; \square$, harmonic field $1 \times 10^{11} \mathrm{~W} / \mathrm{cm}^{2}$, relative phase $\delta=\pi$.

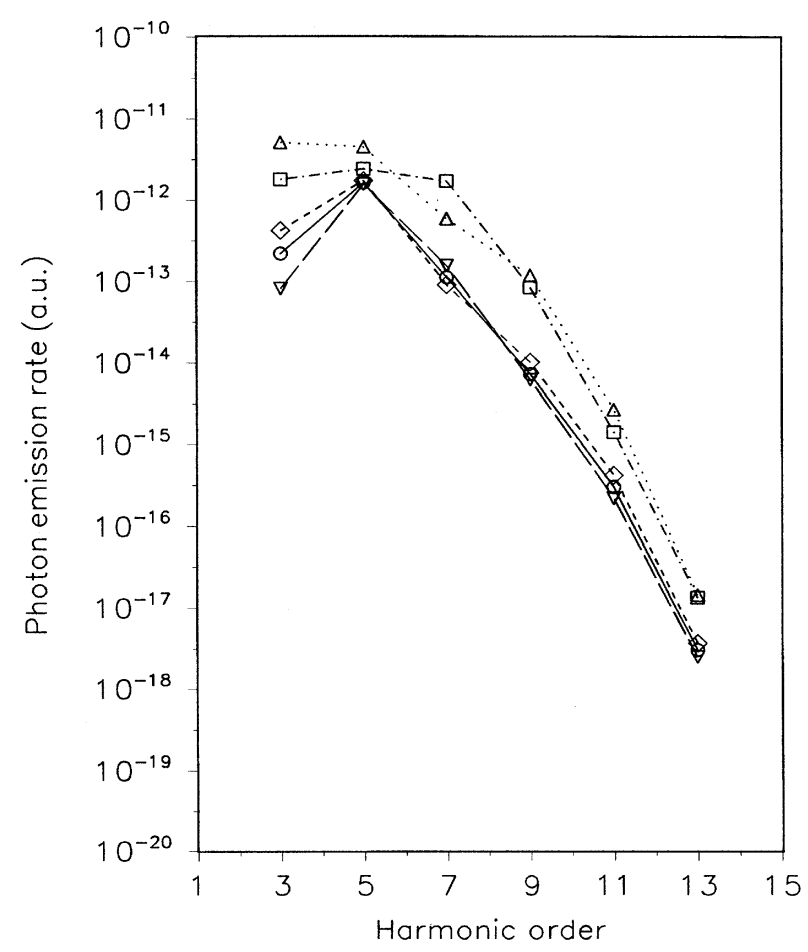

FIG. 2. Harmonic-generation rates (in a.u.) for the fundamental frequency field $532 \mathrm{~nm}$, intensity $5 \times 10^{13} \mathrm{~W} / \mathrm{cm}^{2}$, and its third harmonic. $\bigcirc$, one-color results (fundamental frequency field only); $\diamond$, harmonic field $5 \times 10^{9} \mathrm{~W} / \mathrm{cm}^{2}$, relative phase $\delta=0 ; \nabla$, harmonic field $5 \times 10^{9} \mathrm{~W} / \mathrm{cm}^{2}$, relative phase $\delta=\pi ; \triangle$, harmonic field $5 \times 10^{11} \mathrm{~W} / \mathrm{cm}^{2}$, relative phase $\delta=0 ; \square$, harmonic field $5 \times 10^{11}$ $\mathrm{W} / \mathrm{cm}^{2}$, relative phase $\delta=\pi$. 


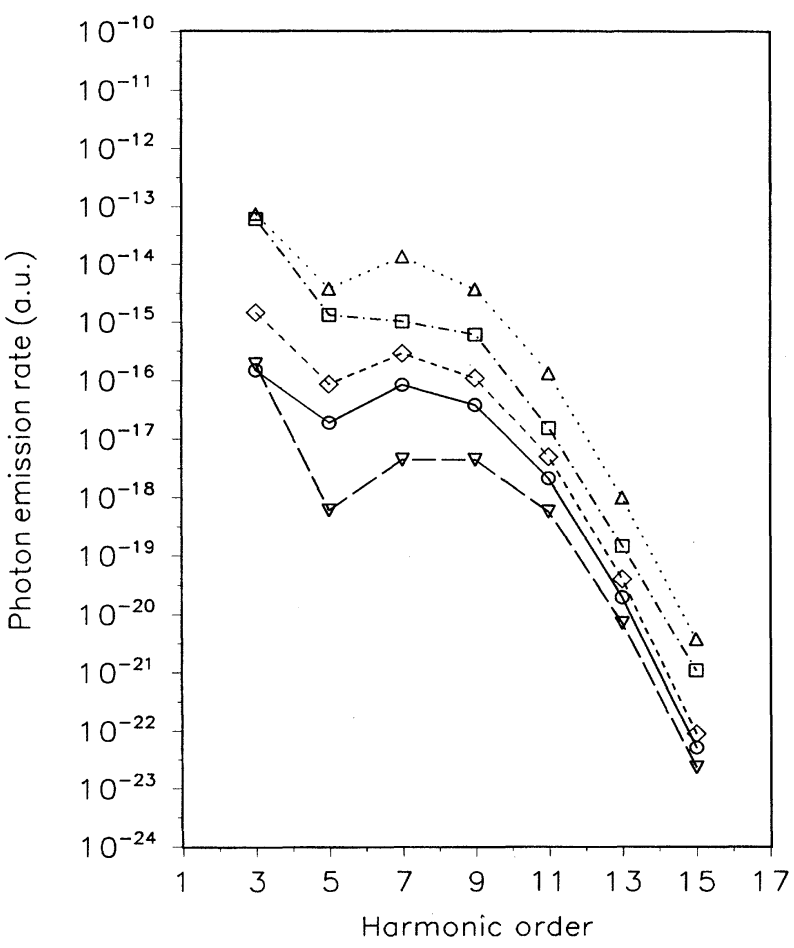

FIG. 3. Harmonic-generation rates (in a.u.) for the fundamental frequency field $775 \mathrm{~nm}$, intensity $1 \times 10^{13} \mathrm{~W} / \mathrm{cm}^{2}$, and its third harmonic. $\bigcirc$, one-color results (fundamental frequency field only); $\diamond$, harmonic field $1 \times 10^{9} \mathrm{~W} / \mathrm{cm}^{2}$, relative phase $\delta=0 ; \nabla$, harmonic field $1 \times 10^{9} \mathrm{~W} / \mathrm{cm}^{2}$, relative phase $\delta=\pi ; \Delta$, harmonic field $1 \times 10^{11} \mathrm{~W} / \mathrm{cm}^{2}$, relative phase $\delta=0 ; \square$, harmonic field $1 \times 10^{11}$ $\mathrm{W} / \mathrm{cm}^{2}$, relative phase $\delta=\pi$.

continuum via the tunneling mechanism. After creation those electrons can return to the nucleus and emit photons during the collision process. The higher the energy of the electron, the higher the frequency of the photon emitted. On the other hand, the energy of the electron depends on the time when the electron was created. Thus, for the fundamental frequency field $F=F_{1} \cos (\omega t)$, the phase $\omega t=0$ (the strongest instantaneous field $F=F_{1}$ ) corresponds to the lowest-energy electrons, whereas the phase $\omega t$ close to $\pi / 2$ corresponds to the highest-energy electrons. However, in the latter case the potential barrier for tunneling is very broad, so the probability of production of high-energy electrons (and, consequently, the probability of radiation of high-energy photons) is small.

Now let us consider how the admixture of a rather weak third-harmonic field can influence this picture. For the phase $\omega t=0$ we have, according to Eq. (19),

$$
F=F_{1}+F_{2}(\delta=0), \quad F=F_{1}-F_{2}(\delta=\pi) .
$$

As one can see, the relative phase $\delta=0$ makes the instantaneous field stronger, whereas the relative phase $\delta=\pi$ makes it weaker. Since the width of the potential barrier depends on the field strength (the weaker the field, the broader the barrier), one can expect enhancement of production of loworder harmonics for $\delta=0$ compared with $\delta=\pi$. Now let the

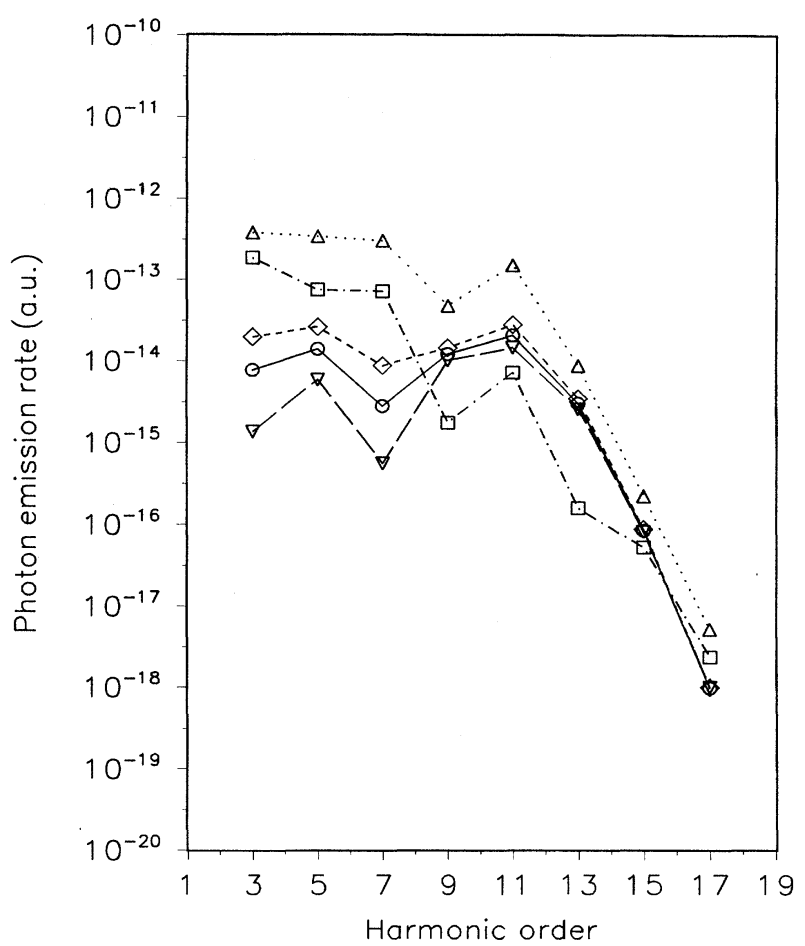

FIG. 4. Harmonic-generation rates (in a.u.) for the fundamental frequency field $775 \mathrm{~nm}$, intensity $3 \times 10^{13} \mathrm{~W} / \mathrm{cm}^{2}$, and its third harmonic. $\bigcirc$, one-color results (fundamental frequency field only); $\diamond$, harmonic field $3 \times 10^{9} \mathrm{~W} / \mathrm{cm}^{2}$, relative phase $\delta=0 ; \nabla$, harmonic field $3 \times 10^{9} \mathrm{~W} / \mathrm{cm}^{2}$, relative phase $\delta=\pi ; \triangle$, harmonic field $3 \times 10^{11} \mathrm{~W} / \mathrm{cm}^{2}$, relative phase $\delta=0 ; \square$, harmonic field $3 \times 10^{11}$ $\mathrm{W} / \mathrm{cm}^{2}$, relative phase $\delta=\pi$.

field phase be $\omega t=\pi / 2+\Delta, \Delta \ll 1$. Instead of Eq. (26) we have for the instantaneous field strength

$$
\begin{aligned}
& F=-\Delta\left(F_{1}-3 F_{2}\right), \quad(\delta=0), \\
& F=-\Delta\left(F_{1}+3 F_{2}\right), \quad(\delta=\pi) .
\end{aligned}
$$

In contrast with the case $\omega t=0$, the instantaneous field is stronger for the relative phase $\delta=\pi$ and weaker for $\delta=0$. This means that the production of high-order harmonics is more effective for the relative phase $\delta=\pi$ than for $\delta=0$.

Certainly, the above consideration cannot explain all the features of HG spectra. However, it gives a qualitative picture that in general is confirmed by our calculations. As one can see, better satisfaction is achieved for the weak thirdharmonic field (100 times weaker than the fundamental frequency field) where the one-color HG rates lie in between the two-color results with $\delta=\pi$ and 0 , respectively. For the stronger third-harmonic field (10 times weaker than the fundamental frequency field) the two-color HG rates for both $\delta=\pi$ and 0 are generally larger than that for the one-color field; however, the relation between the $\delta=\pi$ and 0 cases, according to the semiclassical theory described above, still holds.

\section{CONCLUSION}

In this paper we have presented a nonperturbative Floquet study of HG by two-color laser fields. We used the theory of 
HG based on the quantum-mechanical treatment of the atom and classical treatment of the radiation. The wave functions used by this procedure are computed by means of the nonHermitian Floquet theory and complex scaling, generalized pseudospectral discretization technique. The results obtained in the present paper are for the single-atom HG; we did not take into account the propagation of the radiation in the media. The relation of the single-atom HG rates to the experimental observations is discussed elsewhere (see, e.g. [13]).

The results for the HG spectrum show the following general features. First, the HG rates for two-color laser fields are generally much larger than the rates for the one-color HG, when the third-harmonic field intensity is 10 times smaller than that of the fundamental frequency field. For very weak harmonic field (intensity 100 times smaller than that of the fundamental frequency field) the opposite situation takes place if the relative phase $\delta=\pi$; in this case the one-color results lie in between the two-color results for the relative phases $\delta=0$ and $\pi$. Second, the HG rates manifest a strong dependence on the relative phase between the two fields. For the first few generated harmonics, the largest rates are ob- served for the phase $\delta=0$ and the smallest for $\delta=\pi$. However, for the higher generated harmonics the picture is different: the largest $\mathrm{HG}$ rates correspond to $\delta=\pi$ and the smallest ones correspond to $\delta=0$. This means that the decrease of $\mathrm{HG}$ rates with their number is slower for $\delta=\pi$ than for $\delta=0$.

There is a close analogy between the present results for HG and our previous results for above-threshold multiphoton detachment by two-color laser fields [3]. It confirms our conclusion made in Ref. [3] that the features described above have a general kinematic nature related to the motion of the electron in the two-color laser fields and do not depend qualitatively on the atomic potential (except for the resonance phenomena).

\section{ACKNOWLEDGMENT}

This work was supported by the Division of Chemical Sciences, Office of Basic Energy Sciences of the U.S. Department of Energy.
[1] For two-color ATI experiments, see, for example, D. W. Schumacher, F. Weihe, H. G. Muller, and P. H. Bucksbaum, Phys. Rev. Lett. 73, 1344 (1994); Y. Yian, C. Chen, and D. S. Elliott, ibid. 69, 2353 (1992).

[2] For theoretical studies of two-color ATI processes, see, for example, K. J. Schafer and K. C. Kulander, Phys. Rev. A 45, 8026 (1992); R. M. Potvliege and H. G. Smith, J. Phys. B 25, 2501 (1992); M. Pont, D. Proulx, and R. Shakeshaft, Phys. Rev. A 44, 4486 (1991); G. Yao and S. I. Chu, J. Phys. B 25, 363 (1992).

[3] D. A. Telnov, J. Wang, and S. I. Chu, Phys. Rev. A 51, 4797 (1995).

[4] J. Wang and S. I. Chu, Chem. Phys. Lett. 227, 663 (1994).

[5] A. L'Huillier, L. A. Lompre, G. Mainfray, and C. Manus, Adv. At. Mol. Opt. Phys. Suppl. 1, 139 (1992).

[6] S. Watanabe, K. Kondo, Y. Nabekawa, A. Sagisaka, and Y. Kobayashi, Phys. Rev. Lett. 73, 2692 (1994).

[7] M. D. Perry and J. K. Crane, Phys. Rev. A 48, R4051 (1993).

[8] H. Eichmann, S. Meyer, K. Riepl, C. Momma, and B. Wellegehausen, Phys. Rev. A 50, R2834 (1994).

[9] For reviews on generalized Floquet methods for atomic and molecular multiphoton processes in strong fields, see S. I. Chu, Adv. At. Mol. Phys. 21, 197 (1985); Adv. Chem. Phys. 73, 739 (1989).

[10] R. M. Potvliege and R. Shakeshaft, Phys. Rev. A 40, 3061 (1989).

[11] For numerical integration of the time-dependent Schrödinger equation for the treatment of high-order HG, see, for example, (a) J. L. Krause, K. J. Schafer, and K. C. Kulander, Phys. Rev. A 45, 4998 (1992); (b) T. F. Jiang and S. I. Chu, ibid. 46, 7322 (1992).

[12] S. I. Chu, K. Wang, and E. Layton, J. Opt. Soc. Am. B 7, 425 (1990); G. Bandarage, A. Maquet, and J. Cooper, Phys. Rev. A 41, 1744 (1990).

[13] A. L'Huillier, K. J. Schafer, and K. C. Kulander, J. Phys. B 24, 3315 (1991).

[14] See, for example, B. Sundaram and P. W. Milonni, Phys. Rev. A 41, 6571 (1990).

[15] L. D. Landau and E. M. Lifshitz, Classical Theory of Fields (Pergamon, New York, 1975).

[16] A. Messiah, Quantum Mechanics (North-Holland, Amsterdam, 1965).

[17] T. S. Ho, S. I. Chu, and J. V. Tietz, Chem. Phys. Lett. 96, 464 (1983); T. S. Ho and S. I. Chu, J. Phys. B 17, 2101 (1984).

[18] For reviews on complex scaling transformation, see B. R. Junker, Adv. At. Mol. Phys. 18, 208 (1982); W. P. Reinhardt, Annu. Rev. Phys. Chem. 33, 223 (1982).

[19] J. Wang, S. I. Chu, and C. Laughlin, Phys. Rev. A 50, 3208 (1994).

[20] D. C. Sorensen, SIAM J. Matrix Anal. Appl. 13, 357 (1992).

[21] K. Burnett, V. C. Reed, J. Cooper, and P. L. Knight, Phys. Rev. A 45, 3347 (1992).

[22] P. B. Corcum, Phys. Rev. Lett. 71, 1994 (1993); K. C. Kulander, K. J. Schafer, and J. L. Krause, in Super-Intense LaserAtom Physics, edited by B. Piraux, A. L'Huillier, and K. Rzazewski (Plenum, New York, 1993), p. 95. 